\section{Apprendre à} connaître les patients hospitalisés en oncologie et leur famille: vers une amélioration continue
de la qualité

\author{
par Gail M. Macartney, Gail Stone, \\ Margaret B. Harrison, Ian D. Graham \\ et Catherine E. De Grasse
}

\section{Abrégé}

Étant donnés l'ampleur globale du cancer, l'état actuel des ressources consacrées aux soins de santé et l'activisme politique des survivants du cancer et de leur entourage, il est de plus en plus évident que les pourvoyeurs de services locaux se doivent d'examiner et d'évaluer les soins qu' ils dispensent. On a utilisé un devis qualitatif afin de savoir dans quelle mesure l'établissement hospitalier répondait aux besoins des patients pendant leur séjour, selon ces mêmes patients et leur entourage. Une entrevue semi-structurée a permis de recueillir les commentaires directs d'un échantillon accidentel de 19 patients en oncologie et de membres de leur famille.

$L$ 'analyse qualitative des données a permis de déceler les huit facettes des soins qui importaient aux patients en oncologie et à leur famille. Ces facentes sont les suivantes: communication entre les patients et les prestataires de soins, disponibilité des prestataires et préoccupations vis-à-vis des compressions dans le domaine des soins, coordination et contimuité des soins, soins et confort physiques, soins psychosociaux, sensibilité des prestataires, compétence des prestataires, et enfin, environnement physique de l'établissement.

$L$ 'article met en valeur les défis que doivent relever les personnes atteintes de cancer et leur famille tout au long de leur périple à travers le système de soins de santé. Les répondants appuient les interventions utilisées à bon escient par les soignants tout en traçant. dans leurs grandes lignes, les domaines où une amélioration est nettement désirable

\section{Introduction}

Au cours de sa vie, un(e) résident(e) du Canada sur trois développera une forme quelconque de cancer (Institut national du cancer du Canada [INCC], 1997). De plus, la population vieillit et la fréquence du cancer augmente avec l'âge (Ministère de la Santé de l'Ontario, [MSO], 1993). La prise en charge d'une population âgée présentant des besoins particuliers devient une question de santé de plus en plus aiguë et pressante. Le cancer est un grave problème de santé à la fois pour ceux qui en sont atteints, leur famille et la collectivité.

Gail M. Macartney, Inf, MSc(A), OCN, est infirmière clinicienne-éducatrice; Gail Stone, Inf, oeuvre au Service des soins palliatifs et Catherine E. De Grasse, Inf, $\mathrm{MScN}, \mathrm{OCN}$, est infirmière clinicienne spécialisée en oncologie; toutes travaillent à

l'Hôpital Civic d'Ottawa. Margaret B. Harrison, Inf, candidate au doctorat, est infirmière clinicienne spécialisée,

Recherche et Évaluation et chercheure en chef, Unité d'épidémiologie clinique; Ian D. Graham, MA, PhD, est sociologue clinique et chercheur associé, Unité d'épidémiologie clinique, Institut de recherche Loeb, Hôpital Civic d'Ottawa.
Souvent, le diagnostic de cancer est le point de départ d'une condition chronique, de longue durée. Les personnes touchées entrent alors dans une relation à vie avec le système des soins de santé pour un aspect ou pour un autre de la maladie. Habituellement, le traitement du cancer met en oeuvre des modalités faisant appel à plusieurs disciplines et offertes dans divers types d'établissements (Conkling, 1989; Ferrell et O'Neil-Page, 1993). C'est la raison pour laquelle les patients en oncologie courent souvent le risque de recevoir des soins fragmentés qui ne répondent pas à leurs besoins complexes médicaux, sociaux et psychosociaux (Conkling, 1989; Fletcher, O'Malley, Fletcher et al., 1984; Lauria, 1991).

Ainsi, on prévoit l'augmentation de la fréquence du cancer au cours des vingt prochaines années (MSO, 1994) et parallèlement, celle de la complexité de la prestation des soins et du nombre d'organismes et de secteurs impliqués dans la prestation des services à la population concernéc.

Le présent rapport documente une phase de l'évaluation des besoins relative aux soins dispensés aux patients en oncologie hospitalisés dans un établissement hospitalier d'enseignement en milieu urbain. Il a pour but de fournir une information générale en matière d'évaluation des soins. Cette étude s'inscrivait au sein du programme d'amélioration continue de la qualité.

\section{Phase méthodologie}

\section{Participants à l'étude}

On a rassemblé, sur une période de trois mois, un échantillon accidentel de personnes diagnostiquées de cancer et admises dans l'une ou l'autre de huit unités de soins aux hospitalisés; on leur a demandé de donner leurs commentaires sur les soins qu'elles avaient reçus. On a également invité les membres de la famille et les proches ày participer. On a interviewé un total de 19 patients et de 13 proches.

\section{Méthode de collecte de données}

L'intervieweuse a demandé aux patients et aux membres de la famille s'ils étaient prêts à consacrer 15 à 20 minutes de leur temps à discuter de leur hospitalisation. L'intervieweuse les a prévenus qu'elle

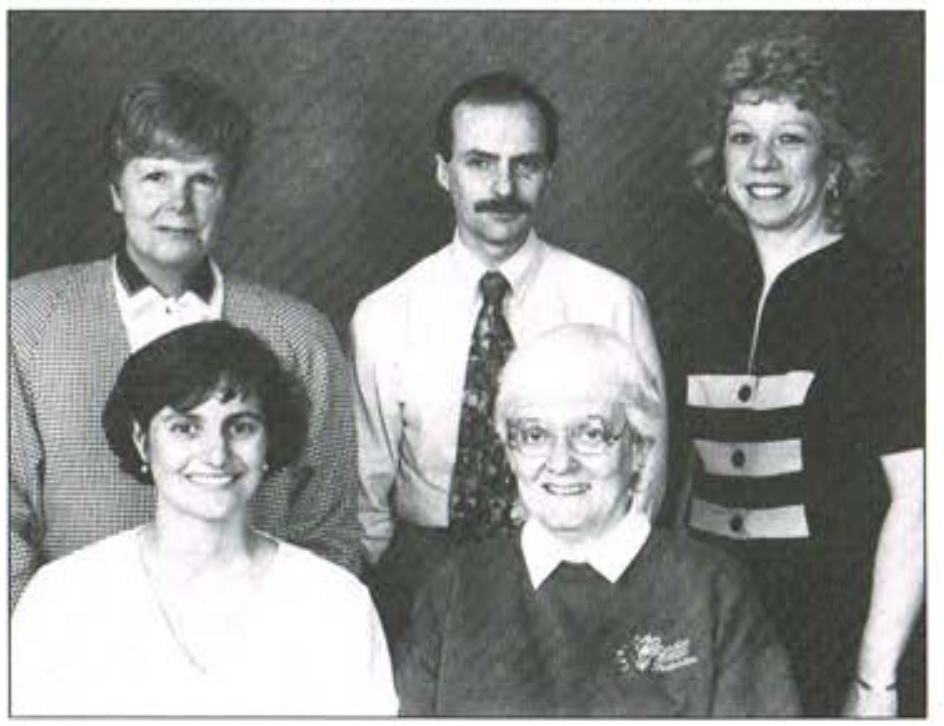

Première rangée, de gauche à droite: Gail Macartney et Gail Stone. Deuxième rangée, de gauche à droite: Margaret Harrison, Ian Graham et Catherine De Grasse 
désirait savoir si leurs besoins étaient satisfaits ou non en vue d'améliorer la prestation des soins. Elle les a aussi assurés du caractère confidentiel de l'entrevue.

Deux infirmières en oncologie ont employé une approche normalisée pour diriger les entrevues semi-structurées. Les entrevues, qui duraient entre 15 et 20 minutes, se sont déroulées dans la chambre des patients pour des raisons pratiques et par souci de confidentialité. Les entrevues n'ont pas été enregistrées mais les infirmières y prenaient des notes détaillées, ce qui a permis de reconstituer les entrevues dès qu'elles étaient terminées. Celles-ci se fondaient sur les questions clés suivantes: (a) Votre séjour à l'hôpital s'est-il déroulé selon vos attentes? (b) Qu'est-ce qui vous a plu? (c) $\mathrm{Y}$ a-t-il des domaines où vous estimez qu'on n'a pas répondu à vos besoins? (d) Y a-t-il autre chose que vous aimeriez nous signaler qui pourrait aider l'hôpital à dispenser de meilleurs soins aux personnes atteintes de cancer?

\section{Analyse qualitative}

On a effectué une analyse qualitative de contenu des données recueillies. Cette analyse de contenu est une stratégie inductive mettant essentiellement en jeu l'examen, la comparaison et la catégorisation des données. Ce type d'analyse permet d'identifier les thèmes présents dans les données (Altheide, 1987; Crabtree et Miller, 1992; Strauss, 1987). Les données se rapportant à chaque entrevue ont été saisies sur un programme de traitement de texte. Les notes prises tout au long d'une entrevue ont reçu un numéro de référence particulier ne comportant aucune information permettant d'identifier l'auteur des propos. On a ensuite remis ces notes aux deux infirmières chercheures et aux deux chercheurs de l'unité d'épidémiologie clinique afin qu'ils en fassent le codage indépendamment les uns des autres. Une fois que les quatre chercheurs ont eu attribué leurs propres codes aux catégories ou thèmes qui se dégageaient des entrevues, ils se sont réunis et se sont concertés sur les unités de signification et leur définition. Puis ils se sont servis du traitement de texte spécialisé Nota Bene (version 4) pour indexer les notes d'entrevue en fonction des unités de signification sur lesquelles ils s'étaient entendus auparavant. Ils ont ensuite récupéré tous les passages associés à une unité de signification donnée, les ont examinés afin de déterminer le bien-fondé de leurs attributions de cette unité. Le cas échéant, ils ont assigné de nouveaux codes en s'efforçant de parvenir à un consensus. IIs ont fait une nouvelle indexation des notes et ont refait la vérification des passages associés aux unités de signification afin de garantir l'uniformité de leur démarche. Enfin, ils ont ré-indexé le tout pour produire les données qui sont à la base du présent rapport. Dans la présente étude, ce sont donc les passages textuels (unités textuelles), et non pas les répondants, qui constituent les unités d'analyse.

\section{Résultats}

Le tableau 1 résume les types de cancers dont sont atteints les membres de l'échantillon. Ces derniers comprenaient 13 patients de sexe féminin et 6 patients de sexe masculin âgés de 38 à 85 ans (pour

Tableau 1 Caractéristiques de l'échantillon

\section{Cancer primaire Nombre de patients}

$\begin{array}{ll}\text { Cancer du sein } & 5 \\ \text { Cancer du côlon } & 3 \\ \text { Leucémie } & 3 \\ \text { Cancer du poumon } & 3 \\ \text { Lymphome } & 2 \\ \text { Cancer du col utérin } & 1 \\ \text { Cancer de l'ovaire } & 1 \\ \text { Mélanome multiple } & 1\end{array}$

un âge moyen de 60 ans) ainsi que 13 membres de la famille.

L'analyse qualitative a révélé huit facettes des soins de santé qui importaient aux patients en oncologie et à leur entourage. Les commentaires, présentés ici dans l'ordre décroissant de leur fréquence, concernaient les points suivants: (a) communication entre les patients et les prestataires de soins ( 50 commentaires faits par 14 personnes), (b) disponibilité des prestataires et préoccupations vis-à-vis des compressions dans le domaine des soins (47 commentaires faits par 15 personnes), (c) coordination et continuité des soins (45 commentaires faits par 14 personnes), (d) soins et confort physiques ( 41 commentaires faits par 16 personnes), (e) soins psychosociaux (33 commentaires faits par 16 personnes), (f) sensibilité des prestataires ( 31 commentaires faits par 13 personnes), (g) compétence des prestataires ( 15 commentaires faits par 7 personnes) et enfin, (h) environnement physique de l'établissement ( 13 commentaires faits par 9 personnes). On présente ci-dessous un résumé de chacune de ces facettes, chaque résumé étant précédé de citations représentatives des commentaires effectués par les patients et leurs proches.

\section{Communication entre les patients et les prestataires de soins} Avant que je réalise ce qui se passait, $j$ 'ai vu une infirmière technicienne devant moi. Elle portait un masque, de grosses lunettes et un tablier aux couleurs bizarres. Elle portait également un tube en carton sur lequel était écrit "Danger biologique" accompagné d'un symbole qui ressemblait à une chauve-souris. Tout cela me rappelait l'Ilalloween et les fantômes. J'étais terrifié. Ensuite, j'ai vu l'infirmière retirer une énorme seringue de ce tube. Vous parlez d'avoir peur! Et tout cela 24 heures seulement après qu' on $m$ 'ait dit qu' il ne me restait peut-être plus que six jours à vive. Habillée et masquée comme elle l'est, portant de grosses lunettes et un tube annonçant "Danger biologique". I infirmière véhicule un message mortel. Je me retrouve en terre étrangère. Expliquez-moi ce que tout cela signifie. Poturquoi ces choses-là sont-elles nécessaires? Expliquez-moi la procédure de $A$ à Z. (Patient de 60 ans souffrant de leucémie aiguè myéloblastique)

Dans I'ensemble, les docteurs se sont montrés attentifs et attentionnès. Mais il y a des fois où ils semblent ne pas écouter ce que j'ai à dire. Parfois je sens que je n'arrive pas à placer un seul mot. Je sais qu'ils sont tous très occupés, mais je crois que cela m'aiderait s'ils prenaient le temps de $s$ 'asseoir ne serait-ce qu'une minute et d'écouter mes inquiétudes. (Patiente de 56 ans souffrant d'un cancer du poumon épidermoïde)

L'étude a mis en relief trois catégories distinctes de questions relatives à la communication. Les patients désiraient: (a) des renseignements et des explications, (b) la chance de pouvoir parler aux prestataires de soins et d'interagir avec eux, et (c) être écoutés par les prestataires de soins.

Recherche d'information et désir qu'on leur explique les choses Les patients souhaitent qu'on les renseigne sur leur condition, leur traitement et le pronostic. Ils se sentent frustrés lorsqu'on ne leur explique pas les choses. Par contre, lorsqu'ils reçoivent des explications, les patients sentent qu'ils contrôlent mieux ce qui leur arrive. Cela permet de réduire l'incertitude et le stress.

Pouvoir parler aux prestataires de soins et interagir avec eux Les patients veulent avoir l'occasion de parler avec leurs soignants. Ils étaient nombreux à assimiler cette absence de communication entre eux et les soignants au fait d'être traités de manière impersonnelle ou "à titre d'objets". En l'absence de dialogue, ils décrivaient les prestataires de soins comme étant "froids et distants".

Être à l'écoute des patients Les patients veulent qu'on les écoute et surtout qu'on les entende. D'après eux, les prestataires de soins répondaient à leurs besoins lorsqu'ils faisaient l'effort de les écouter. Les patients ont parlé de la frustration qu'ils ressentaient lorsque les soignants ne voulaient pas entendre ce qu'ils disaient. 
Disponibilité des prestataires et préoccupations vis-à-vis des compressions dans le domaine des soins

Il n'y a pas suffisamment d'infirmières. Elles ont trop à faire

On comprend qu'il y a des urgences mais l'autre jour.

l'infirmière a mis bien longtemps avant de venir. On a su plus

tard que quelqu'un souffrait d'un ulcère qui saignait

abondamment. Elles font ce qu'elles peuvent. (Le fils d'une

patiente de 67 ans souffrant de leucémie lymphoíde chronique)

Il s'agit donc ici du thème de la disponibilité des pourvoyeurs de soins et des inquićtudes vis-à-vis des compressions telles que vues depuis la perspective des patients. Les patients voulaient que les prestataires de soins soient immédiatement disponibles pour répondre à leurs besoins bien qu'ils réalisent que les soignants étaient très occupés ou surmenés. Le manque de disponibilité du personnel était attribué aux compressions budgétaires affectant les soins de santé. Ils ont dégagé une stratégie importante permettant de faire face à cette situation: engager la participation de la famille et des amis au niveau de la prestation des soins. Le thème à l'étude a été subdivisé en quatre catégories: (a) la perception du personnel soignant comme étant insensible aux besoins des patients, (b) l'apparence du personnel qui est très occupé ou même surmené, (c) la conscience des compressions, (d) le besoin de faire participer la famille aux soins.

Satisfaction des besoins Les patients appréciaient le fait que les soignants satisfassent rapidement à leurs besoins. II ressort clairement que les patients étaient mécontents lorsque leurs besoins n'étaient pas satisfaits dans des délais raisonnables.

Apparence du personnel qui est très occupé ou même surmené La moitié des patients ont indiqué qu'ils étaient vivement conscients du fait que les infirmières étaient très occupées et surmenées. Dans certains de leurs commentaires, les patients en concluaient que les soignants étaient trop occupés pour fournir la quantité ou le type de soins qu'ils auraient aimé recevoir.

Conscience des compressions Les patients et leur entourage attribuaient au déclin des ressources les compromis qu'ils percevaient en matière de niveau ou de qualité des soins. Non seulement les patients jugeaient que les compressions avaient une incidence directe sur les soins physiques qu'ils recevaient, mais encore ils croyaient qu'elles avaient une incidence indirecte à cause du stress qu'elles font naître chez les prestataires de soins.

Besoin de faire participer la famille aux soins Les commentaires d'un nombre relativement faible de répondants semblaient indiquer que, selon cux, les compressions érodaient sérieusement les soins aux patients. Ces commentaires révélaient que les membres de la famille estimaient qu'ils devaient participer activement aux soins physiques dispensés à leur proche puisque le personnel soignant de l'établissement hospitalier était dans I'impossibilité de répondre aux besoins de ce dernier.

\section{Coordination et continuité des soins}

J'étais tellement heureux de voir la même infirmière la denxième nuit que j'ai passée à l'hôpital. J'espérais qu'elle viendrait car elle connaissait déjà mes exigences et je n'avais pas besoin de hui redire tous les détails de mon cas. (Patient de 47 ans atteint d'un cancer du côlon et de métastases hépatiques)

$L$ 'an dernier, $j$ 'ai dî me rendre au service des urgences pour une transfusion de sang (pendant la semaine, je peux aller à l'unité des procédures spéciales pour cela et là, ils s'y connaissent vraiment). Mais aux urgences, ils ne savaient que faire dans mon cas; il n'y avait personne qui savait comment ouvrir mon cathéter Hickman. Cela aurait dî prendre cinq mimutes mais ça a pris des heures et des heures. (Patiente de 38 ans atteinte d'un cancer du sein et de métastases pulmonaires et cérébrales)

Le thème de la coordination se rapporte à l'organisation des soins et à la structure du système des soins de santé au sein même de I'hôpital. II reflète l'interaction et l'interconnexion des divers services et composantes du système de soins. Le manque de coordination se traduisait avant tout par l'obligation d'attendre. Les patients veulent des soins coordonnés. Dans le cas de patients gravement malades, l'attente est frustrante et pour certains, exaspérante. Un bon nombre de patients étaient parfaitement conscients que leur espérance de vie était brève. Ils ne souhaitaient pas passer trois quarts d'heure à répéter leur histoire ou attendre des heures durant dans les couloirs d'un établissement hospitalier.

Les patients accordent donc une grande importance à la continuité des soins. Par continuité des soins, ils entendent avoir les mêmes prestataires (infirmières et docteurs) qui gèrent et coordonnent les soins et ils tiennent à ce que ces prestataires de soins apprennent à les connaître.

\section{Soins et confort physiques}

On ne nous donne plus de frictions dans le dos parce que les infirmières ont trop à faire. Cet aspect des soins me manque beaucoup. (Patiente de 56 ans atteinte d'un cancer du poumon épidermoïde)

Les patients étaient nombreux à mentionner les soins et le confort physiques. Les commentaires portaient majoritairement sur les besoins physiques qui, d'après les patients, n'étaient pas satisfaits; ainsi, ils voudraient qu'on s'occupe d'eux plus fréquemment la nuit, qu'on les aide à trouver des positions plus confortables, qu'on leur donne des frictions dans le dos et qu'on leur serve des repas plus appétissants. Lorsque I'on étudie ces préoccupations apparemment mineures à la lumière des raisons du séjour hospitalier de ces patients, on comprend mieux l'importance qu'elles revêtent pour les patients et leur entourage.

Par contre, plusieurs patients ont signalé leur satisfaction générale relative aux soins reçus. Il est intéressant de noter que, bien que peu de patients indiquaient qu'ils étaient heureux de recevoir leurs médicaments à l'heure donnée, aucun d'entre eux ne s'est plaint de pas avoir reçu d'analgésiques.

\section{Soins psychosociaux}

Les infirmières étaient conscientes de tout ce qui ne touche pas directement au traitement médical et étaient armées pour y faire face, Elles savaient répondre aux larmes. Ces infirmières étaient compétentes et chevronnées. Elles savaient être à l'écoute et comprendre les situations. Je crois que le personnel médical a tendance à ne tenir compte que des statistiques des patients telles que les mumérations globulaires et la température. Il ne semble pas s'intéresser à la personne humaine dans son ensemble. Les aspects psychologiques, émotionnels et spirituels se perdent en route. (Patient de 60 ans souffrant de lencémie aiguë myéloblastique)

Nombreux sont les patients qui ont offert des commentaires axés sur les soins psychosociaux. Ils y mettaient en relief l'importance que revêt le soutien psychosocial pour les patients et leur famille. Ces commentaires étaient pour la plupart positifs. Toutefois, un tiers des commentaires suggéraient que les besoins sociaux et psychologiques des patients n'entraient pas du tout en ligne de compte ou qu'on y répondait pas d'une manière qui satisfasse les attentes des patients. Ces derniers indiquaient qu'ils s'attendaient à ce que les pourvoyeurs de soins répondent aussi bien à leurs besoins psychosociaux qu'à leurs besoins physiques.

\section{Sensibilité des prestataires}

Les enfants et moi, on l'accompagnait quand on l'a admis à l'hôpital vers 2 h 30 du matin. Nous avons attendu en dehors de sa chambre pendant que cinq infirmières l'aidaient à $s$ 'installer. En sortant, chacune de ces infirmières nous a salué et nous a parlé aux uns comme aux autres. Elles nous ont montré où trouver du thé et du jus. Elles nous ont mis à l'aise dès le début. Elles m'ont dit que si je me faisais du souci que je ne devrais pas hésiter à appeler les infirmières au poste. $L$ 'infirmière ne s'est pas contentée de dire "Est-ce que ça va?" à mon mari, elle me l'a demandé à moì aussi. (Épouse d'un patient de 42 ans atteint d'un lymphome hodgkinien en phase terminale) 
Ce quit me bouleverse le plus, ce sont les docteurs qui

discutent de leurs cas sur le pas de ma porte. J'entends souvent ce qu'tls disent. Cela me fait peur car je me demande toujours si c'est de moi qu'ils parlent ou de quelqu'un d'autre. (Patient de 62 ans atteint d'un mélanome multiple)

On a réuni sous ce thème les commentaires qui faisaient ressortir la sensibilité des prestataires face aux besoins et préoccupations des patients ou bien leur manque de sensibilité à cet égard. Un certain nombre de commentaires indiquaient à quel point les patients se sentaient estimés lorsque les prestataires de soins étaient attentionnés et respectueux. Par exemple, des commentaires illustraient l'impact positif qu'ont chez les patients de "petites" choses comme le fait de se présenter ou de prendre le temps de parler avec l'entourage du patient. Dans bien des cas, il suffisait aux soignants d'être courtois et polis pour manifester leur sensibilité.

\section{Compétence des prestataires}

La compétence est une qualité que je recherche chez tous mes soignants. Je désire avoir des infirmières qui savent comment manipuler les machines, les tubes et les médicaments qui m'entourent. Lors de ma première séance de chimio, $j$ 'ai eu la chance d'avoir une infirmière perspicace. Je n'avais pas besoin de me dire "Puis-je faire confiance à cette personne au sujet de toutes ces machines puissantes et potentiellement dangereuses?". En fait, je $n$ 'ai eu que quatre infirmières durant ces quelques jours où j'étais terriblement malade. Cela m'a permis de connaitre chacune d'entre elles et de leur accorder ma confiance. Je n'avais pas besoin de réitérer tous les détails à chaque changement de quart. Je veux être certain que mes infirmières sont compétentes et qu'elles connaissent bien la maladie dont je souffre... Certaines personnes peuvent vous côtoyer pendant de brefs instants et vous inspirer confiance en expliquant ce qu'elles font et en manifestant leur compétence. (Patient de 60 ans souffrant de leucémie aiguē myéloblastique)

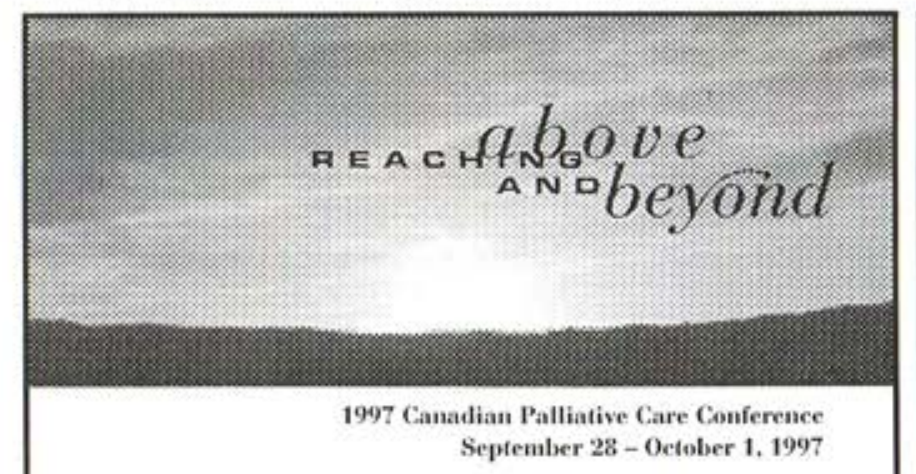

Fall and harest on the Prairies bring uonderful sights, sounds and smells. It is to this setting you are imvited to attend the 1997 Canadian Palliative Car Conference in picturesque Saskatoon - the hub city of the province. The confenence features speakers Mark Miller, Balfour-Monut, Beth Pery and Bouren White. Come lears, shatre, celebrate and meet new friends as ue gather around the theme, "Reaching Above and Beyond."

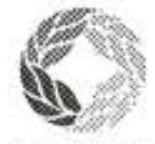

SISKATCHEWAN PALLLATIVE CAIE ASSOCIATION
For more information, please contact:

Sask. Palliative Care Association

III - 230 Avenue R South

Saskatoon, SK SZK $2 Z 1$

phome: 306 .352-25.50

fax: $306.352-3445$

c-mail:cpe97@xdh.sk.co
Les commentaires reflétant la confiance des patients vis-à-vis des prestataires de soins étaient à la fois positifs et négatifs; certains se sentaient en confiance et en sécurité avec les soignants tandis que d'autres éprouvaient des sentiments opposés. Lorsque les patients estimaient que les soignants n'étaient pas compétents, ils n'avaient pas confiance en eux et dans certains cas, avaient même peur d'eux. Pour certains répondants, la compétence des soignants ne se mesure pas seulement en termes d'habileté technique; on s'attend aussi à ce que les soignants soient capables de se charger de l'aspect psychosocial. Le simple fait d'expliquer aux patients les procédures et les approches avait pour résultat d'augmenter la confiance des patients envers leurs soignants. Quelques commentaires suggéraient que, chez les infirmières, un haut niveau de stress se traduisait par une baisse de la compétence.

\section{Environnement physique de l'établissement}

Je ne dormais pas bien à l'hôpital, ce qui fait que j'en sortais fatiguée et affaiblie. C'est un cercle vicieux. Les portes des deux salles de préparation des traitements claquent toute la nuit, je suis cerlaine qu'on pourrait éviter cela. Une fois, on $m$ 'a mise dans une chambre avec une dame qui mourrait du cancer. Celle-ci n'arrêtait pas de gémir, le jour comme la nuit. Elle aurait dâ être dans une chambre individuelle. Si on $n$ 'arrive pas à dormir, je crois que cela retarde le rétablissement et que l'hospitalisation dure plus longtemps. (Patiente de 56 ans atteinte d'un cancer du sein métastatique) Est-ce qu'ils stérilisent les chaises d'aisance entre deux patients? Moi, je ne pense pas. Cela vous tente-t-il d'utiliser une chaise d'aisance sale? (Patiente de 78 ans atteinte d'un cancer du côlon métastatique)

Les répondants ont fourni un certain nombre de commentaires sur l'environnement physique de l'établissement hospitalier; la plupart d'entre eux se rapportaient aux problèmes ou aux inquiétudes des patients face à leur environnement physique. Ils portaient sur la propreté des salles de bains, sur le contrôle de la climatisation (forte chaleur, l'été, dans certaines salles), sur la qualité de l'air qui entraîne

\section{(U oncology nursing distance EDUCATION COURSE CROSS CANCER INSTITUTE}

The Cross Cancer Institute Department of Nursing in Edmonton, Alberta welcomes registered nurses to enroll in a distance education course in oncology nursing.

Study in the comfort of your home with a unique, self-directed education program. We will provide instructor support by telephone, learning modules and access to support materials.

The Oncology Nursing Distance Education Course has been peer reviewed by the Canadian Association of Nurses in Oncology and meets the nursing program criteria for a three-credit senior elective at Athabasca University and at the University of Alberta.

For information and application call:

(403) $432-8746$ 
la sécheresse de la bouche et de la gorge. Les patients ont également mentionné plusieurs facteurs qui affectaient la qualité du sommeil, notamment l'éclairage, le bruit, les courants d'air aux fenêtres et les autres patients.

\section{Incidences sur les soins infirmiers}

Les répondants ont indiqué qu'ils voulaient connaître leur plan de soins, particulièrement l'information concernant le traitement, les procédures et le pronostic. Les patients se sentent valorisés lorsqu'on leur donne la possibilité de discuter de leur plan de traitement avec leurs soignants, et ce, dans une atmosphère calme et détendue. Un grand nombre de patients se sont dit frustrés par le manque de coordination de leurs soins qui se traduisait inévitablement par l'attente. L'utilisation de plans de cheminement critique est une initiative de plus en plus populaire visant à favoriser la prestation de soins rentables répondant aux besoins des patients (Hofmann, 1993). La mise au point de plans de cheminement relatifs à une unité particulière dans le cadre de traitements ou de procédures d'un coût élevé et à grand débit ayant des résultats prévisibles, pourrait faciliter la communication du plan de soins multidisciplinaires aux patients et à leur famille.

Les patients jugeaient que le manque de continuité au niveau des soignants se traduisait par une détérioration au niveau de la continuité des soins. Il ressort que la mise en oeuvre des soins infirmiers intégraux en tant que système de prestation des soins devrait améliorer la continuité au niveau de la relation avec les prestataires de services infirmiers qui est essentielle au développement de la relation thérapeutique (Procter, 1995).

Plusieurs patients ont dégagé des questions de santé pour lesquelles on n'a pas répondu à leurs besoins comme le positionnement ou les frictions dans le dos. Dans certains cas, les membres de la famille et les amis voulaient participer activement à la prestation de ces soins de santé de base. Les infirmières sont à même de faciliter la participation de la famille aux soins physiques en évaluant la volonté des membres à le faire et en se chargeant des besoins d'éducation.

Les aspects des soins dont les patients parlaient le plus positivement avaient trait à la manière dont l'équipe des soins de santé s'occupait d'eux. Les exemples parlent d'infirmières manifestant de la compassion, de l'empathie et une grande bienveillance, de l'importance de se présenter, de reconnaître la présence de la famille et d'avoir un bon sens de l'humour. Les patients et leur famille voulaient qu'on leur fasse sentir qu'ils étaient les bienvenus, qu'on les appelle par leur nom et qu'on les écoute. Les patients désirent qu'on les traite avec respect, empathie et courtoisie. Ils souhaitent qu'on les traite en tant que personnes et non pas en tant que numéros ou cas. Les soins psychosociaux étaient parfois mis en péril par le manque de sensibilité des soignants.

Les besoins identifiés dans l'étude correspondent au paradigme de besoins d'ordre pratique, spirituels, psychosociaux, informationnels, affectifs et physiques du Modèle des soins de soutien (Fondation ontarienne pour la recherche en cancérologie et le traitement du cancer [FORCTC], 1994). L'approche qui permet de répondre aux besoins d'une personne en matière de soins de soutien exige un partenariat avec le patient (FORCTC, 1994, p. 14). II convient d'inclure l'identification des besoins relatifs aux soins de soutien dans les évaluations infirmières continues de ces patients afin qu'on puisse adapter les interventions en vue d'y répondre.

Selon notre étude, il importe que les soignants répondent aux besoins humains fondamentaux en manifestant leur sensibilité dans le cadre des soins dispensés et en faisant appel à leur compétence interpersonnelle. Par "compétence interpersonnelle", on entend la capacité à établir et à maintenir des relations empruntes d'empathie qui facilitent la réalisation d'objectifs précis tout en affirmant la valeur des participants à ces relations (Taylor, 1995). Taylor soutient que les infirmières se sont efforcées de relever les défis scientifiques et techniques inhérents à la pratique professionnelle et que ces efforts se sont traduits par un abaissement de l'excellence traditionnelle de la profession infirmière en matière de compétence interpersonnelle. Les stratégies visant à promouvoir la compétence interpersonnelle incluent les suivantes: une utilisation novatrice du regard, de la parole et du toucher afin de communiquer le respect et de promouvoir le sentiment de valeur des patients; une utilisation appropriée de la présence et de la conversation permettant de manifester son empathie et de recueillir suffisamment d'information au sujet du patient pour personnaliser les soins et défendre efficacement ses droits; une attention responsable et compétente portée aux besoins holistiques des patients afin d'instaurer la confiance et de permettre aux patients d'éprouver le confort qui va de pair avec le sentiment de sécurité (Taylor, 1995).

Les résultats de l'étude mettent en valeur quelques-uns des défis auxquels font face les personnes atteintes du cancer et leur famille tout au long de leur périple à travers le système des soins de santé. Il convient donc de sensibiliser les infirmières s'occupant de telles personnes aux problèmes mis en lumière. La prestation de soins attentionnés qui répondent aux besoins des patients n'est pas une proposition onéreuse.

\section{Bibliographie}

Altheide, D.L. (1987). Ethnographic content analysis. Qualitative Sociology, 10(1), 65-67.

Conkling, V. (1989). Continuity of care issues for cancer patients and families. Cancer, 64, 290-294.

Crabtree, B., Miller, W. (Eds.). (1992). Research methods in primary care (Vol. 3). Newbury Park, CA: Sage.

Ferrell, B.R., O'Neil-Page (1993). Continuity of care. In S. Groenwald, M. Fogge \& C. Yarbro (Eds.), Cancer nursing: Principles and practice (3rd ed.) (pp.1346-1359). Boston: Jones \& Bartlett.

Fletcher, R.H., O'Malley, M.S., Fletcher, S.W., Earp, J.A., Alexander, J.P. (1984). Measuring the continuity and coordination of medical care in a system involving multiple providers. Medical Care, 22(5), 403-411.

Hofmann, P.A. (1993). Critical path method: An important tool for coordinating clinical care. Journal on Quality Improvement, 19, 235-246.

Lauria, M.M. (1991). Continuity of cancer care. Cancer, 67, 1759-1766.

Ministry of Health Ontario. (1994). Life to gain: A cancer strategy for Ontario. Toronto: Queen's Printer for Ontario (Cat \#2226232). Ministry of Health Ontario. (1993). A healthier Ontario: Progress in the '90s. Toronto: Queen's Printer for Ontario (Cat \#4225539).

Institut national du cancer du Canada. Statistiques canadiennes sur le cancer en 1997, Toronto, Canada.

Ontario Cancer Treatment and Research Foundation. (1994, May). Providing supportive care for individuals living with cancer. Toronto, Canada: Author.

Procter, S. (1995). Planning for continuity of care in nursing. Journal of Nursing Management, 3(4), 169-175.

Strauss, A. (1987). Qualitative analysis for social scientists. Cambridge: Cambridge University.

Strauss, A., Corbin, J. (1990). Basics of qualitative research: Grounded theory procedures and techniques. Newbury Park, CA: Sage Publications.

Taylor, C. (1995). Rethinking nursing's basic competencies. Journal of Nursing Care Quality, 9(4), 1-13. 